$\begin{array}{llllll}\text { A C T A } & \text { C H E M I C A } & \text { S C A N D I N A V I C A } & 17 & \text { (1963) } & \text { S } 302-S 307\end{array}$

\title{
Die Isolierung eines kristallinen Antithiaminwirkstoffes aus Karpfeneingeweide
}

\author{
J. C. SOMOGYI und H. KUNDIG
}

Institut für Ernährungsforschung, Rüschlikon-Zürich, Schweiz

\begin{abstract}
Eine Methode zur Isolierung eines kristallinen Antithiaminwirkstoffes aus Karpfeneingeweide wird beschrieben. Die Rohkristalle sind dunkelbraun, prismenförmig und leuchten im polarisierten Licht auf. Sie weisen folgende Zusammensetzung auf: C 60,41\%; H 8,69\%; O 24,52\%; N 7,15\%. Zersetzungspunkt: $250-270^{\circ} \mathrm{C}$. Die Rohkristalle sind im Gegensatz zum Ausgangsmaterial (Rohextrakt, Dialysat) thermostabil. Die I. R.- und U. V.Spektren, sowie andere charakteristische Eigenschaften der Substanz werden mitgeteilt. Auf das Auffinden eines zweiten, noch nicht weiter untersuchten Antithiaminwirkstoffes aus Karpfeneingeweide wird hingewiesen.
\end{abstract}

$\mathrm{U}$

nsere früheren Versuche ${ }^{1-3}$ haben gezeigt, dass die im Rohextrakt und in einem durch Dialyse gereinigten Extrakt enthaltenen Antithiaminwirkstoffe aus Karpfeneingeweide thermolabil und nicht dialysierbar sind. Im elektrischen Feld wandern sie teilweise kathodisch, teilweise verhalten sie sich elektroneutral. Später haben wir ${ }^{4}$ eine weitere Reinigung eines Antithiaminwirkstoffes durch wiederholte Fällungen mit Ammoniumsulfat $\left(\mathrm{AmSO}_{4}\right)$ und beim isoelektrischen Punkt erzielt. In einigen Fällen konnte eine kristalline Substanz isoliert werden. Der genaue Vorgang, welcher zur Bildung dieser Kristalle führt, blieb damals jedoch unabgeklärt.

Wir haben darum vor ca. zwei Jahren diese Versuche wieder aufgenommen. Es gelang, die Reinigungsmethode so weit zu entwickeln, dass der Wirkstoff nunmehr praktisch ausnahmslos als kristalline Substanz erhalten werden kann. Ferner hat sich gezeigt, dass das Karpfeneingeweide, wie früher vermutet, einen zweiten Antithiaminfaktor mit anderen chemischen und physicochemischen Eigenschaften enthält.

\section{METHODIK}

Die Bestimmung des Thiamins erfolgte mit der Thiochrommethode und mit Hilfe eines von uns beschriebenen Fluorimeters $s^{5}$. Um zu vergleichbaren Ergebnissen zu gelangen, wurde die Antithiaminaktivität verschiedener Reinigungsstufen mit einem modifizierten 50 \%-Inaktivierungstest bestimmt und die Aktivität in $\gamma$ inaktiviertem Thiamin/mg Trockensubstanz bzw. Eiweiss ausgedrückt. Bei dieser Methode wird diejenige Menge eines Antithiaminpräparates 
Tabelle 1. Schema der Isolierung eines Antithiaminwirkstoffes aus Karpfeneingeweide. Alle Operationen bei der Reinigung erfolgten, falls nichts anderes vermerkt, bei ca. $+4^{\circ} \mathrm{C}$.

\section{Eingeweide (KE)}

1: 2 mit $10 \% \mathrm{NaCl}$ in Turmix verklein., einfrieren mit Aceton/Trockeneis, 48 Std. stehen lassen bei -14 bis $-20^{\circ} \mathrm{C}$

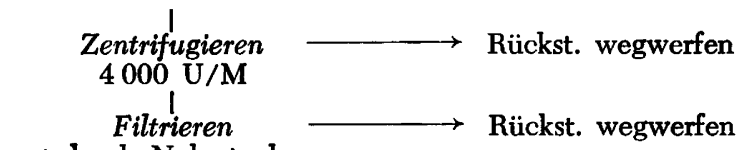

durch Nylontuch

Dialyse

fliess. Wasser 4 Std. + dest. Wasser

$\mathrm{pH} 7.0$ bis Elektrolyt frei

Zentrifugieren

$1 \times 4000 \mathrm{U} / \mathrm{M}$

$1 \times 14000 \mathrm{U} / \mathrm{M} \longrightarrow$ Rückst. wegwerfen

Dialysat (EDZ)

$3 \times \mathrm{AmSO}_{4} \mathrm{Fällung}(\mathrm{pH} \mathrm{5.0)} \longrightarrow$ Uberstehendes wegw. Am-Präcipitat jeweils in $\mathrm{H}_{2} \mathrm{O}$ pH 7.0 lösen

Am-Präcipitat III

\section{Dialyse}

fliess. Wasser 4 Std. + dest. Wasser bis $\mathrm{SO}_{4}$ frei

Zentrifugieren

$1 \times 4000 \mathrm{U} / \mathrm{M} \mathrm{pH} 7.0 \longrightarrow$ Rückst. wegwerfen

Uberstehendes zentrifugieren

$14000 \mathrm{U} / \mathrm{M}$ pH 7.0

Uberstehendes

fällen bei $\mathrm{pH} 5.0$

Zentrifugieren

Niederschlag bei pH 7.0 lösen

Präcipitat I

Wiederholen so lange Akt. steigt

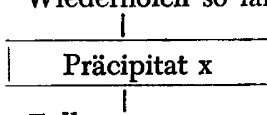

Fällung

Ionenstärke red. $0,15 \rightarrow 0.05, \mathrm{pH} 6,8 \rightarrow 5,2$

Zentrifugieren

$$
\frac{\text { Niederschlag }}{+\mathrm{H}_{2} \mathrm{O} \text { und bei } 4^{\circ} \mathrm{C}}
$$$$
1-3 \mathrm{~W} \text {. stehen lassen }
$$

Niederschlag (Rstd.)

lösen bei $\mathrm{pH} 8.0$

Rückstand I 
festgestellt, die bei einer Vorlage von $100 \gamma$ Thiamin/20 ml Versuchsgemisch $50 \gamma$ zu inaktivieren vermag. Daraus wird berechnet, wieviel $\gamma$ Thiamin durch $1 \mathrm{mg}$ Substanz inaktiviert wird.

\section{VERSUCHE}

Der Reinigungsvorgang ist schematisch in Tab. 1 zusammengefasst. Alle Operationen erfolgten, falls nichts anderes vermerkt, bei ca. $+4^{\circ} \mathrm{C}$.

Der Rohextrakt des Karpfeneingeweides (Herz, Niere, Milz und Leber) wird dialysiert, zentrifugiert und anschliessend dreimal mit Ammoniumsulfat gefällt. Das Präparat wird bis zur völligen Freiheit von Sulfat dialysiert; anschliessend erst bei einer Tourenzahl von 4000 und dann bei $14000 \mathrm{U} / \mathrm{Min}$. bei $4^{\circ} \mathrm{C}$ zentrifugiert. Es bildet sich beim hochtourigen Zentrifugieren ein Niederschlag (Bezeichnung: Rückstand), der eine mittlere Antithiaminaktivität, jedoch andere chemische bzw. physicochemische Eigenschaften aufweist als die nachfolgend beschriebene Substanz (Präcipitat).

Der Niederschlag (Rückst.) wandert im elektrischen Feld nämlich bei $\mathrm{pH} 8.6$ kathodisch (das Präcipitat wandert nicht), weist einen N-Gehalt von 4,6\% (Präcipitat 7,1\% N) auf, ist in Wasser erst bei $\mathrm{pH} 8$ und darüber löslich (das Präcipitat löst sich schon bei $\mathrm{pH} 7,0$ ). Die Isolierung und weitere Aufklärung der chemischen Natur dieses zweiten Antithiaminwirkstoffes soll später durchgeführt werden.

Der grössere Teil der Antithiaminaktivität ist in der überstehenden Lösung enthalten. Diese wird nun beim isoelektrischen Punkt ( $\mathrm{pH} \mathrm{5.0)}$ gefällt. Es entsteht ein Präcipitat, welches nach Zentrifugieren bei $\mathrm{pH} 7.0$ wieder gelöst wird. Dieser Vorgang wird so oft wiederholt bis die Aktivität des Präcipitats nicht mehr steigt. Auf diese Weise gelangt man zu einem Präparat, das eine 60-90 mal grössere Antithiaminaktivität aufweist (auf Trockengewicht bezogen) als der Rohextrakt.

Bei unseren früheren Versuchen ist der Wirkstoff aus der aktivsten Fraktion in 2-3 Fällen auskristallisiert, aber die Bedingungen, die zur Kristallisation der Substanz führten, blieben - wie erwähnt - unbekannt.

Es wurde hierauf auf verschiedene Weise versucht, die Kristalle zu erhalten. So z. B. durch weitere Reinigung des Präcipitates mit Hilfe einer DEAE Ionenaustauscher-Zellulosekolonne gemäss Sober und Mitarbeiter ${ }^{6-8}$, durch Fällung des aktivsten Präcipitates mit Ammoniumsulfat usw. Keine dieser Methoden hat die gewünschten Ergebnisse gezeitigt.

Zum Erfolg führte eine sog. "Euglobulin-Fällung" der aktivsten Fraktion. Bei der Dialyse fällt im Dialysenschlauch, der das gelöste Präcipitat enthält, durch sukzessive Verringerung der Ionenkonzentration und gleichzeitiges Ansäuren der Aussenflüssigkeit ein Niederschlag aus. Dieser wird zentrifugiert und nach Zugabe von Wasser in einem Uhrglas in hochkonzentrierter Form bei $4^{\circ} \mathrm{C}$ stehen gelassen. Nach 1-3 Wochen Aufbewahrung im Kühlschrank fallen die Antithiaminwirkstoff-Kristalle aus. Die Ausbeute ist gering. Sie variiert je nach der Aktivität des Präcipitates zwischen 8-25\%.

Die Erhöhung der Antithiaminaktivität während dieses Reinigungsvorgangs ist aus der folgenden Abbildung (Abb. 1) ersichtlich. Wie daraus zu entnehmen ist, weist die kristalline Substanz eine ca. 100-200 mal grössere Antithiaminaktivität als der Rohextrakt auf. 


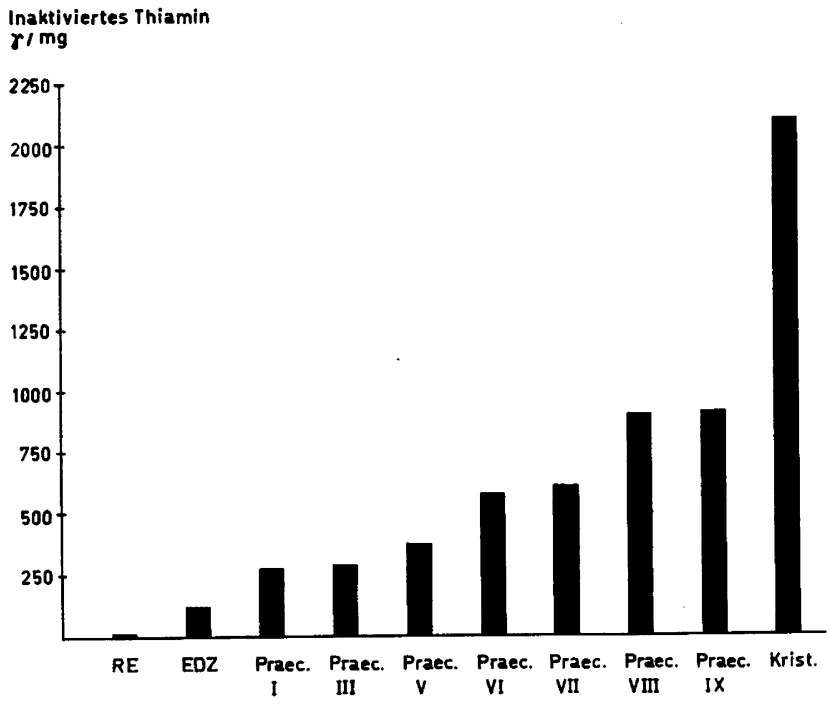

$A b b$. 1. Die Antithiaminaktivität verschiedener Reinigungsstufen. $\mathrm{RE}=$ Rohextrakt, $\mathrm{EDZ}=$ Dialysat, Praec. $=$ Praecipitat, Krist. $=$ Rohkristalle (s. auch Tab. 1).

Die Kristalle sind dunkelbraun, prismenförmig und leuchten im polarisierten Licht auf. (Abb. 2). Sie sind im Wasser (bei $\mathrm{pH} 7$ und darüber), in $\mathrm{Na}_{2} \mathrm{CO}_{3}$, in 10 N Ameisensäure, Pyridin, Aethanolamin usw. löslich, in Aethylalkohol, Aether, Aceton unlöslich. Zersetzungspunkt: $260-270^{\circ} \mathrm{C}$.

Die Kristalle sind im Gegensatz zu Ausgangsmaterial (Rohextrakt, Dialysat) thermostabil. Erhitzen auf $100^{\circ} \mathrm{C}$ während 1 Std. verringert die Antithiaminaktivität nicht. Sie sind nicht dialysierbar. Die Mikroanalysen der Rohkristalle ergaben folgendes Resultat: C 60,41\%; H 8,69\%; O 24,52\%; N 7,15\% auf aschenfreie Substanz berechnet. Der Wirkstoff ist frei von Schwefel, Phosphor, Jod, Brom Kobalt, Schwermetallen und von reduzierendem und nicht reduzierendem Zucker. Die Substanz enthält Chlorid und gibt positive Phenolreaktionen.
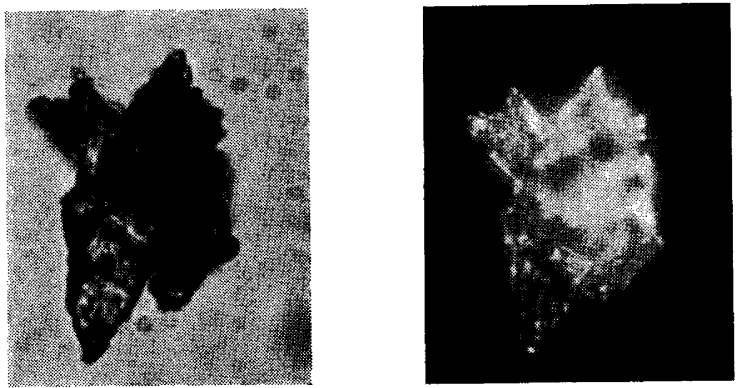

Abb. 2. Kristalle des Antithiaminwirkstoffes links in gewöhnlichem und rechts in polarisiertem Licht. 


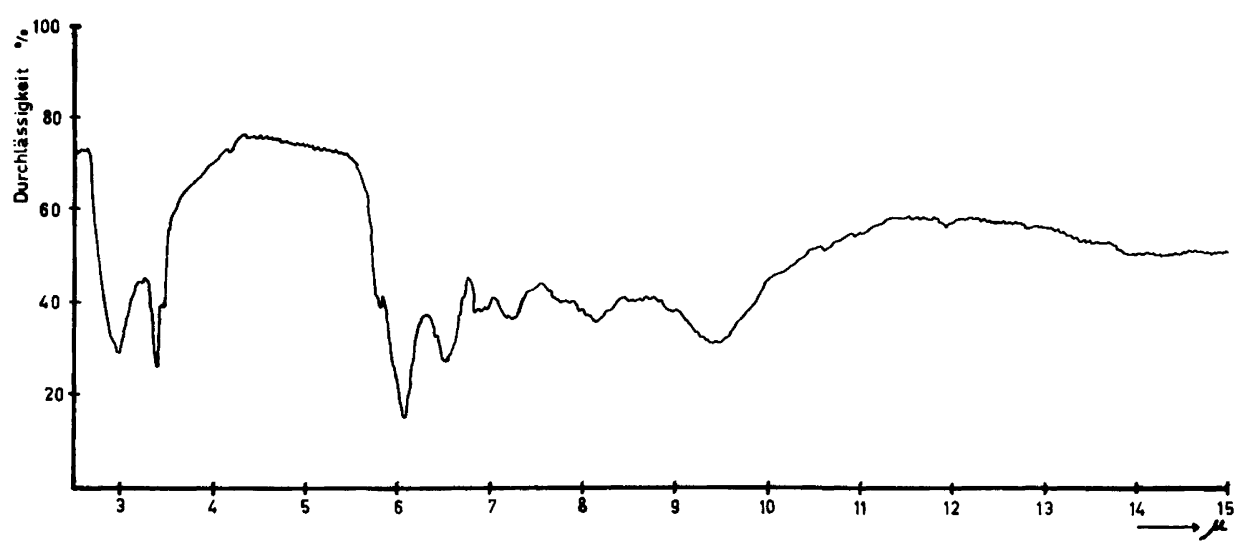

Abb. 3. I. R.-Spektrum des kristallinen Antithiaminwirkstoffes.

Eine Umkristallisation dieser Rohkristalle konnte bis anhin nur in einigen Fällen durchgeführt werden. Die Antithiaminaktivität, Form und Farbe der Kristalle, sowie der Zersetzungspunkt blieb unverändert.

Wie aus der Mikroanalyse hervorgeht, enthält die Substanz nur 7,15 \% N. Daraus folgt, dass dieser Antithiaminwirkstoff kein typisches Eiweiss ist. Es kann sich aber auch nicht um ein Glykosid handeln, da weder in der Substanz, noch im Hydrolysat desselben reduzierende bzw. nicht reduzierende Zucker nachweisbar sind. Die Verbindung muss jedoch ein grösseres Molekül sein, da sie die Dialysenmembrane nicht passiert.

Sowohl die Mikroanalysen, wie auch die mehrmals aufgenommenen I.R.Spektren und die praktisch immer gleich grosse Antithiaminaktivität der Kristalle (2200 - $2400 \gamma / \mathrm{mg}$ Trockengewicht) zeigen, dass die beschriebene Reinigungsmethode ein reproduzierbares Präparat ergibt.

Gemäss I.R.-Spektrum (Abb. 3) handelt es sich bei den Rohkristallen um eine weitgehend einheitliche Substanz, die eine oder mehrere Amidgruppen, sekundäre und tertiäre $\mathrm{OH}-\mathrm{Gruppen}\left(>\mathrm{CH}_{2}+-\underset{\mid}{\mathrm{C}} \mathrm{H}\right)$ und eventuell phenolische $\mathrm{OH}$ Gruppen enthält.

Zum Schluss soll noch erwähnt werden, dass das U. V.-Spektrum der Rohkristalle (gelöst im Wasser, pH 7,0) ein deutliches Maximum bei $398 \mathrm{~m} \mu$ aufweist.

Die weite Aufklärung der chemischen Konstitution dieser Substanz ist in Arbeit.

Dem Schweizerischen Nationalfonds für wissenschaftliche Forschung möchten wir für die gewährte Unterstützung unseren besten Dank aussprechen.

Herrn P. D. Dr. A. Dreiding, der die Aufnahmen der I. R.-Spektra im Organisch-Chemischen Institut der Universität Zürich veranlasst hat, danken wir bestens. Die Mikroanalysen wurden von Herrn E. Thommen, Organisch-Chemische Anstalt der Universität Basel, durchgeführt. 


\section{LITERATUR}

1. Somogyi, J. C. Helv. Physiol. Pharmacol. Acta 7 (1949) C 24. Intern. Z. Vitaminforsch. 21 (1949) 341 .

2. Somogyi, J. C. Helv. Physiol. Pharmacol. Acta 9 (1951) C 79.

3. Somogyi, J. C. Die Antithiaminfaktoren, Hans Huber Verlag, Bern, 1952.

4. Somogyi, J. C. Ergebnisse der med. Grundlagenforschung; Metaboliten und Antimetaboliten, Georg Thieme Verlag, Stuttgart, 1956, S. 157.

5. Somogyi, J. C. und Koller, A. Bull. Schweiz. Akad. Med. Wiss. 14 (1958) 172.

6. Peterson, E. A. und Sober, H. A. J. Am. Chem. Soc. 78 (1956) 751.

7. Sober, H. A., Gutter, F. J., Wyckoff, M. M. und Peterson, E. A. J. Am. Chem. Soc. 78 (1956) 756.

8. Sober, H. A. und Peterson, E. A. Federation Proc. 17 (1958) 1116.

Eingegangen am 22. Februar 1963. 